\title{
Estudio comparativo de facilidades y obstáculos de las mipymes exportadoras en el estado de Guanajuato de México y en la provincia de limón de Costa Rica
}

Comparative study of facilities and obstacles of the exporting mipymes in the state of Guanajuato of Mexico and in the lemon province of Costa Rica

Kenishia Peart-Harris ${ }^{1}$

Peart-Harris, K. Estudio comparativo de facilidades y obstáculos de las mipymes exportadoras en el estado de Guanajuato de México y en la provincia de limón de Costa Rica. Tecnología en Marcha. Vol. 33, especial Movilidad estudiantil. Pág 104-112.

doi) https://doi.org/10.18845/tm.v33i7.5483 


\section{Palabras clave}

Gestión de Calidad; Desempeño Exportador; Exportación; Facilidades; Obstáculos,

\section{Resumen}

Con este estudio se pretende comparar las principales facilidades y obstáculos que enfrentan las mi pymes exportadoras del Estado de Guanajuato, México y de la Provincia de Limón, Costa Rica.

Se intentó identificar si existen similitudes o diferencias entre las facilidades y obstáculos que presentan las Mi pymes exportadoras del Estado de Guanajuato, México y de la Provincia de Limón, Costa Rica.

El estudio va dirigido a todo aquel que exporta o tiene deseos de exportar, ya que les dará herramientas útiles e información para comprender un poco el ámbito de las exportaciones en México y Costa Rica.

Se utilizó el método de a entrevista para recabar la información necesaria con una muestra no probabilística o dirigida por conveniencia. Se obtuvieron los siguientes resultados por indicador:

En limón se encuentran micro, pequeñas y medianas empresas mientras que en Guanajuato solo se encuentran pequeñas y medianas empresas. En Guanajuato y en Limón las empresas pertenecen a los sectores industrial y comercial. Tienen la similitud de que exportan vegetales.

Las pymes en el estado de Guanajuato comenzaron sus actividades de exportación tiempo antes que las mipymes de Limón. Las empresas cuentan con capital propio y presentan similitudes y diferencias en cuanto a retos al exportar, normas de calidad y los mecanismos de control de procesos que utilizan.

\section{Keywords}

Quality Management; Export Performance; Export; Facilities; Obstacles; MSMEs.

\section{Abstract}

This study aims to compare the main facilities and obstacles faced by exporting MSMEs in the State of Guanajuato, Mexico and the Province of Limón, Costa Rica.

An attempt was made to identify if there are similarities or differences between the facilities and obstacles presented by the exporting Mipymes of the State of Guanajuato, Mexico and the Province of Limón, Costa Rica.

The study is aimed at anyone who exports or has a desire to export, as it will give them useful tools and information to understand a little the scope of exports in Mexico and Costa Rica.

The interview method was used to gather the necessary information with a non-probabilistic or convenience-driven sample. The following results were obtained by indicator:

In lemon, there are micro, small and medium-sized companies, while in Guanajuato there are only small and medium-sized companies. In Guanajuato and Limón the companies belong to the industrial and commercial sectors. They have the similarity that they export vegetables.

SMEs in the state of Guanajuato began their export activities some time before the Limón MSMEs. The companies have their own capital and present similarities and differences in terms of export challenges, quality standards and the process control mechanisms they use. 


\section{Introducción}

El presente estudio describió aspectos relacionados con la gestión de la calidad y el desempeño exportador de las mipymes en el Estado de Guanajuato, México y en la Provincia de Limón, Costa Rica.

El tipo de investigación que se aplica es la descriptiva-exploratoria-cuantitativa.

Con esta investigación se intentará identificar si en materia de exportación las mipymes cuentan con facilidades u obstáculos y será útil para que los dueños o encargados de las mipymes puedan poner en práctica mecanismos de control de procesos, utilicen herramientas de mejora y apliquen las normas de gestión de la calidad (si no lo estuvieran haciendo) para asegurar el éxito de su empresa desde la calidad. Su utilidad puede ser social, económica y administrativa.

La investigación se lleva a cabo a partir del mes de Enero 2017 (durante el 2107 se recopiló la información en México) hasta Mayo del 2018 (durante el 2018 se recopiló la información en Costa Rica), en donde se recopila, da formato y analiza la información de las mipymes.

Se planteó como principal objetivo del proyecto el comparar las principales facilidades y obstáculos que enfrentan las mipymes exportadoras del Estado de Guanajuato, México y las de Provincia de Limón, Costa Rica.

\section{Métodos}

\section{Enfoque de la investigación}

Es cuantitativo porque usa la recolección de datos con base en la medición numérica y el análisis estadístico, para establecer patrones de comportamiento [1].

Se inició el proceso con una investigación exploratoria que se desarrolló en dos etapas, la primera etapa consistió en la revisión de literatura relacionada con las mipymes exportadoras; también se consultaron las bases de datos. La segunda fase consistió en una serie de entrevistas con los jefes, encargados y dueños de las mipymes del estado de Guanajuato y de la provincia de Limón.

\section{Diseño de la investigación}

Es no experimental con un alcance exploratorio y descriptivo. Exploratoria porque se indaga el tema desde una perspectiva innovadora y descriptiva porque se considera el fenómeno estudiado y sus componentes [1].

La temporalidad es transversal, porque recopila información en un momento dado, permitiendo comparar la situación actual en dos escenarios diferentes: Guanajuato y Limón.

\section{Unidad de análisis}

Las mipymes del estado de Guanajuato y de la provincia de Limón. 


\section{Población y muestra}

Está conformada por las mipymes del Estado de Guanajuato y de la Provincia de Limón. Se decidió seleccionar una muestra no probabilística o dirigida por conveniencia. Los individuos empleados en la investigación se seleccionaron porque están fácilmente disponibles, no porque hayan sido seleccionados mediante un criterio estadístico. La muestra en el estado de Guanajuato, México fue de 10 empresas y en la provincia de Limón, Costa Rica fue de 5 empresas.

\section{Variables de la investigación}

A partir de los objetivos específicos se determinaron las variables más relevantes del estudio, las cuales se presentan en el cuadro 1.

\section{Cuadro 1. Variables del Estudio}

\begin{tabular}{|c|c|c|}
\hline Variable & Definición conceptual & Definición operacional \\
\hline $\begin{array}{l}\text { Tamaño de } \\
\text { empresa }\end{array}$ & $\begin{array}{l}\text { El tamaño o dimensión de la empresa quiere expresar el } \\
\text { mayor o menor volumen de cada una de las magnitudes } \\
\text { que sirven para explicar lo que es y lo que hace la unidad } \\
\text { económica [2]. }\end{array}$ & $\begin{array}{l}\text { - Micro: número de empleados } \leq 10 \\
\text { - Pequeña: } 10 \leq \# \text { de empleados } \leq 30 \\
\text { - Mediana: } 30 \leq \# \text { de empleados } \leq 100\end{array}$ \\
\hline Sector & $\begin{array}{l}\text { División de la actividad económica de un país en función de } \\
\text { la propiedad de las empresas o del tipo de actividad [3]. }\end{array}$ & $\begin{array}{l}\text { - Industrial } \\
\text { - Comercial }\end{array}$ \\
\hline $\begin{array}{l}\text { Desempeño } \\
\text { exportador }\end{array}$ & $\begin{array}{l}\text { El desempeño exportador generalmente se mide con el } \\
\text { índice de éxito exportador el cual es de uso reciente en } \\
\text { las mipymes, procura encontrar una medida sintética que } \\
\text { permita cuantificar el grado de éxito exportador de cada } \\
\text { empresa en particular, con lo cual se construye un ranking } \\
\text { que muestra el mayor o menor grado de aprovechamiento } \\
\text { de los mercados externos por parte de las pequeñas y } \\
\text { medianas empresa del país [4]. }\end{array}$ & $\begin{array}{l}\text { - Continuidad exportadora } \\
\text { - Dinamismo exportador } \\
\text { - Diversificación de mercados } \\
\text { - Condiciones de acceso a los mercados }\end{array}$ \\
\hline Obstáculo & Situación o hecho que impide el desarrollo de una acción [5]. & - Instrumento aplicado \\
\hline Facilidades & $\begin{array}{l}\text { Condiciones especiales que permiten lograr algo o alcanzar } \\
\text { un fin con menor esfuerzo [6]. }\end{array}$ & - Instrumento aplicado \\
\hline Calidad & $\begin{array}{l}\text { Es el grado en el que un grupo de características inherentes } \\
\text { cumplen con los requisitos. }\end{array}$ & $\begin{array}{l}\text { - Existencia de normas } \\
\text { - Mecanismos de control } \\
\text { - Cultura de calidad } \\
\text { - Herramientas para la mejora }\end{array}$ \\
\hline
\end{tabular}

\section{Análisis comparativo mipymes de Guanajuato vs mipymes de Limón}

En esta sección se muestra un análisis comparativo entre los resultados más relevantes obtenidos en el estado de Guanajuato, México y en la provincia de Limón, Costa Rica. 
Cuadro 10. Comparación de resultados

\begin{tabular}{|c|c|c|c|c|}
\hline Indicador & $\begin{array}{c}\text { Resultados de } \\
\text { Guanajuato, México }\end{array}$ & $\begin{array}{l}\text { Resultados de Limón, } \\
\text { Costa Rica }\end{array}$ & Comparación & Similitud \\
\hline $\begin{array}{l}\text { Tipo de } \\
\text { empresa }\end{array}$ & $\begin{array}{l}\text { Más de la mitad de las } \\
\text { empresas entrevistadas } \\
\text { están en la clasificación } \\
\text { de pequeñas empresas, } \\
\text { ya que poseen menos } \\
\text { de } 40 \text { trabajadores. }\end{array}$ & $\begin{array}{l}\text { Más de la mitad } \\
\text { de las empresas } \\
\text { entrevistadas están } \\
\text { en la clasificación de } \\
\text { pequeñas empresas, } \\
\text { ya que poseen menos } \\
\text { de } 40 \text { trabajadores. }\end{array}$ & $\begin{array}{l}\text { En limón se encuentran } \\
\text { micro, pequeñas y medianas } \\
\text { empresas mientras que } \\
\text { en Guanajuato solo se } \\
\text { encuentran pequeñas y } \\
\text { medianas empresas. }\end{array}$ & \\
\hline $\begin{array}{l}\text { Sector al que } \\
\text { pertenece }\end{array}$ & $\begin{array}{l}\text { Poco menos del } 50 \\
\% \text { de empresas se } \\
\text { dedican a ambas } \\
\text { actividades: creación } \\
\text { de productos y su } \\
\text { comercialización. }\end{array}$ & $\begin{array}{l}\text { Más del } 55 \% \text { de } \\
\text { empresas se dedican } \\
\text { a ambas actividades: } \\
\text { creación de productos } \\
\text { y su comercialización. }\end{array}$ & $\begin{array}{l}\text { El mayor porcentaje de } \\
\text { empresas que se encuentran } \\
\text { en ambos sectores } \\
\text { productivos se encuentra } \\
\text { más consolidado en el } \\
\text { mercado, ya que pueden } \\
\text { dedicarse a los dos sectores } \\
\text { y asegurar su éxito en cada } \\
\text { uno de ellos. }\end{array}$ & \\
\hline $\begin{array}{l}\text { Productos } \\
\text { que exportan }\end{array}$ & $\begin{array}{l}\text { La mayor parte de las } \\
\text { empresas se dedican a } \\
\text { exportar y /o producir } \\
\text { materiales de acabados } \\
\text { y vegetales y hortalizas. }\end{array}$ & $\begin{array}{l}\text { La mayor parte de } \\
\text { las empresas de } \\
\text { dedican a exportar } \\
\text { y/o producir piña y } \\
\text { banano. }\end{array}$ & $\begin{array}{l}\text { Ambos sectores exportan } \\
\text { productos diferentes, pero } \\
\text { se haya similitud en la } \\
\text { exportación de vegetales. }\end{array}$ & \\
\hline $\begin{array}{l}\text { Años de } \\
\text { exportar }\end{array}$ & $\begin{array}{l}\text { La mayor parte de } \\
\text { la empresas poseen } \\
\text { de } 15 \text { a } 20 \text { años } \\
\text { de experiencia en } \\
\text { exportación }\end{array}$ & $\begin{array}{l}\text { La mayor parte de } \\
\text { las empresas poseen } \\
\text { menos de } 10 \text { años } \\
\text { de experiencia en } \\
\text { exportación. }\end{array}$ & $\begin{array}{l}\text { Se halló una diferencia } \\
\text { marcada en la experiencia } \\
\text { de exportación de las } \\
\text { empresas, ya que más del } \\
25 \% \text { de las empresas en } \\
\text { Guanajuato poseen de } 15 \text { a } \\
20 \text { años de experiencia en } \\
\text { exportación lo que indica } \\
\text { que han atravesado por los } \\
\text { diferentes cambios en cuanto } \\
\text { a aspectos propios de la } \\
\text { exportación y han tenido } \\
\text { que adaptarse al cambiante } \\
\text { mercado mientras que más } \\
\text { del 35\% de las empresas en } \\
\text { Limón tienen menos años } \\
\text { de realizar actividades de } \\
\text { exportación. }\end{array}$ & \\
\hline $\begin{array}{l}\text { Inversión } \\
\text { extranjera }\end{array}$ & $\begin{array}{l}\text { Un mayor porcentaje } \\
\text { de las empresas no } \\
\text { cuentan con inversión } \\
\text { extranjera. }\end{array}$ & $\begin{array}{l}\text { Un mayor porcentaje } \\
\text { de las empresas no } \\
\text { cuentan con inversión } \\
\text { extranjera }\end{array}$ & $\begin{array}{l}\text { Existe la similitud de que } \\
\text { la mayoría de las empresas } \\
\text { cuentan con capital propio, } \\
\text { lo que se puede deber a que } \\
\text { las mismas son empresas } \\
\text { familiares. }\end{array}$ & \\
\hline
\end{tabular}




\begin{tabular}{|c|c|c|c|c|}
\hline Indicador & $\begin{array}{c}\text { Resultados de } \\
\text { Guanajuato, México }\end{array}$ & $\begin{array}{l}\text { Resultados de Limón, } \\
\text { Costa Rica }\end{array}$ & Comparación & Similitud \\
\hline $\begin{array}{l}\text { Retos para } \\
\text { exportar }\end{array}$ & $\begin{array}{l}\text { Para la mayor parte } \\
\text { de las empresas el } \\
\text { reto más grande a la } \\
\text { hora de exportar es } \\
\text { el posicionamiento } \\
\text { competitivo a nivel } \\
\text { internacional, ya } \\
\text { que en el mercado } \\
\text { internacional se } \\
\text { encuentran miles } \\
\text { de empresas que se } \\
\text { dedican a las mismas } \\
\text { actividades. } \\
\text { Una minoría de la } \\
\text { muestra considera } \\
\text { como retos a la } \\
\text { hora de exportar las } \\
\text { instalaciones físicas, } \\
\text { las transacciones } \\
\text { internacionales } \\
\text { y las habilidades } \\
\text { transculturales. } \\
\text { Una de la empresas } \\
\text { considera que no } \\
\text { posee retos, ya que } \\
\text { los responsables } \\
\text { adquirieron la empresa } \\
\text { cuando ya estaba } \\
\text { consolidada, los } \\
\text { retos los tuvieron los } \\
\text { creadores dela empresa } \\
\text { (sus padres). }\end{array}$ & $\begin{array}{l}\text { Para la mayor parte } \\
\text { de las empresas el } \\
\text { reto más grande a } \\
\text { la hora de exportar } \\
\text { es el marketing } \\
\text { internacional, porque } \\
\text { dependiendo de } \\
\text { la manera en que } \\
\text { promocionen sus } \\
\text { productos así será } \\
\text { la respuesta del } \\
\text { consumidor final. } \\
\text { Una minoría considera } \\
\text { un reto las alianzas } \\
\text { estratégicas. } \\
\text { Para la totalidad } \\
\text { de las empresas } \\
\text { las habilidades } \\
\text { transculturales y las } \\
\text { instalaciones físicas no } \\
\text { representan un reto a } \\
\text { la hora de exportar. }\end{array}$ & $\begin{array}{l}\text { El mayor reto para exportar } \\
\text { en el estado de Guanajuato } \\
\text { es el posicionamiento } \\
\text { competitivo internacional } \\
\text { mientras que en Limón es el } \\
\text { marketing internacional. } \\
\text { Los menores retos para } \\
\text { exportar de las empresas en } \\
\text { el estado de Guanajuato son } \\
\text { las instalaciones físicas, las } \\
\text { transacciones internacionales } \\
\text { y las habilidades } \\
\text { transculturales mientras que } \\
\text { en las empresas de Limón } \\
\text { consideran que son las } \\
\text { alianzas estratégicas. }\end{array}$ & \\
\hline
\end{tabular}




\begin{tabular}{|c|c|c|c|c|}
\hline Indicador & $\begin{array}{c}\text { Resultados de } \\
\text { Guanajuato, México }\end{array}$ & $\begin{array}{l}\text { Resultados de Limón, } \\
\text { Costa Rica }\end{array}$ & Comparación & Similitud \\
\hline $\begin{array}{l}\text { Normas de } \\
\text { calidad }\end{array}$ & $\begin{array}{l}\text { La mayoría de las } \\
\text { empresas utilizan las } \\
\text { normas de gestión de } \\
\text { calidad ISO } 9001 . \\
\text { La minoría de las } \\
\text { empresas utiliza de las } \\
\text { empresas utilizan las } \\
\text { normas de gestión de } \\
\text { calidad ISO9004, las } \\
\text { normas de seguridad y } \\
\text { salud en el trabajo ISO } \\
45001 \text { y las normas de } \\
\text { seguridad alimentaria } \\
\text { HACCP ISO } 22000 \text { (food } \\
\text { safety) } \\
\text { Dos de las empresas } \\
\text { utilizan normas } \\
\text { adicionales que son las } \\
\text { ISO } 28000 \text { (seguridad } \\
\text { de la cadena de } \\
\text { suministro) y la ISO } \\
17025 \text { (requisitos que } \\
\text { deben cumplir los } \\
\text { laboratorios de ensayo } \\
\text { y calibración). }\end{array}$ & $\begin{array}{l}\text { Debido a que las } \\
\text { empresas de Limón } \\
\text { se dedican a la } \\
\text { comercialización de } \\
\text { frutas y vegetales } \\
\text { utilizan las normas de } \\
\text { seguridad y salud en } \\
\text { el trabajo ISO } 45001 \text { y } \\
\text { las normas de gestión } \\
\text { ambiental ISO } 14000 . \\
\text { La totalidad de las } \\
\text { empresas utiliza las } \\
\text { normas de gestión de } \\
\text { calidad ISO9004 y las } \\
\text { normas de seguridad } \\
\text { alimentaria HACCP ISO } \\
22000 \text { (food safety). }\end{array}$ & $\begin{array}{l}\text { En ambos lugares las } \\
\text { empresas utilizan las normas } \\
\text { ISO 9001, la ISO } 22000 \text { (food } \\
\text { safety), la ISO } 14000 \text { y la ISO } \\
45001 . \\
\text { La diferencia es que en } \\
\text { el estado de Guanajuato } \\
\text { también utilizan las ISO } \\
28000 \text { y la ISO } 17025 .\end{array}$ & \\
\hline $\begin{array}{l}\text { Mecanismos } \\
\text { de control de } \\
\text { procesos }\end{array}$ & $\begin{array}{l}\text { La mayoría de las } \\
\text { empresas utilizan los } \\
\text { Procedimientos de } \\
\text { producción. } \\
\text { La menor parte de } \\
\text { las empresas utilizan } \\
\text { la medición de } \\
\text { desperdicios (dándole } \\
\text { poca importancia } \\
\text { al manejo de los } \\
\text { desperdicios que a lo } \\
\text { largo acarrearan costos } \\
\text { para la empresa). }\end{array}$ & $\begin{array}{l}\text { La mayoría de las } \\
\text { empresas utilizan el } \\
\text { Manual de calidad, } \\
\text { los Instructivos de } \\
\text { trabajo y la medición } \\
\text { de desperdicios como } \\
\text { mecanismos de } \\
\text { control de procesos. }\end{array}$ & $\begin{array}{l}\text { En el estado de } \\
\text { Guanajuato las empresas } \\
\text { utilizan mayormente } \\
\text { los Procedimientos de } \\
\text { producción mientras que en } \\
\text { Limón las empresas utilizan } \\
\text { los manuales de calidad, los } \\
\text { Instructivos de trabajo y la } \\
\text { medición de desperdicios } \\
\text { como mecanismos de control } \\
\text { de procesos. }\end{array}$ & \\
\hline
\end{tabular}

Fuente: Elaboración propia con base en los resultados de la aplicación de las encuestas.

\begin{tabular}{|l|l|}
\hline \multicolumn{2}{|c|}{ Código de similitud } \\
\hline Similar \\
\hline Con semejanzas y diferencias \\
\hline Diferentes \\
\hline
\end{tabular}




\section{Conclusiones}

En Guanajuato las facilidades que se identifican son la Selección de mercados internacionales, la Ubicación de la empresa y la adquisición de proveedores y los obstáculos son las políticas gubernamentales (burocracia), las tasas de interés altas y financiamiento, las limitaciones de producción y la ubicación de las empresas.

En Limón las facilidades que se identifican son la adquisición de proveedores (por la ubicación geográfica), las buenas prácticas, la selección de nuevos mercados internacionales y la ubicación de las empresas. Los obstáculos son las tasas de interés y financiamiento, las políticas gubernamentales (burocracia) y la ubicación de las empresas.

En Limón se encuentran micro, pequeñas y medianas empresas mientras que en Guanajuato solo se encuentran pequeñas y medianas empresas.

El mayor porcentaje de empresas que se encuentran en ambos sectores productivos, ambos sectores exportan productos diferentes, pero se haya similitud en la exportación de vegetales y hortalizas.

Como la mayor parte de las empresas mipymes de Guanajuato se dedican a exportar y /o producir materiales de acabados y vegetales y hortalizas nos habla de los gustos de la población, ella se inclina más a la adquisición de los acabados de construcción y presenta los vegetales como parte de su dieta regular y en Limón por ser el caribe de Costa Rica, cuenta con las condiciones climáticas idóneas para producir frutas y vegetales frescos de las que muchos países carecen.

Entre el estado de Guanajuato y la provincia de Limón existe la similitud de que la mayoría de las empresas pymes y mipymes exportadoras cuentan con capital propio lo que se puede deber a que las mismas son empresas familiares y se caracterizan por contar con capital propio.

El mayor reto para exportar en el estado de Guanajuato es el posicionamiento competitivo a nivel internacional mientras que en Limón es el marketing internacional.

No existe una política pública orientada a reducir los obstáculos y aprovechar las facilidades para las pymes en el estado de Guanajuato y en la provincia de Limón a la hora de exportar por lo tanto en las recomendaciones se plantean algunos elementos a considerar para dicha política.

\section{Cuadro 13: Sugerencias de elementos a considerar.}

- Agilización de trámites gubernamentales para la exportación, para eliminar la burocracia, que es la que hace que muchas mipymes no realicen actividades de exportación.

- Crear una campaña informativa que sirve para que cada dueño de mipymes conozca a fondo los requerimientos gubernamentales que deben cumplir para exportar.

- Realizar capacitaciones para la certificación de los productores nacionales en normas internacionales (ISO), en mecanismos de control de procesos y en normas de calidad.

- Crear una base de datos completa, accesible y actualizada de las empresas mipymes exportadoras, ya que el país maneja la información a nivel de PROCOMER y Cámara de comercio de manera privada, por lo que es imposible acceder a ella. Como por ejemplo la del SIEM (Sistema de Información Empresarial Mexicano) que es una base de datos empresarial pública.

- Crear programas universitarios con énfasis en la creación de mipymes y en la promoción de la exportación de los productos que nos caracterizan como provincia y como país 


\section{Recomendaciones}

- Explotar la ubicación estratégica de las empresas para ampliar la producción y adquirir nuevos clientes.

- Continuar enlazado a los proveedores actuales y conseguir más proveedores de calidad para que los tiempos de entrega puedan ser los necesarios para cumplir con los requerimientos de los clientes.

- Minimizar la cantidad de papeleo que se debe presentar para ser apto para exportar.

- Formular políticas públicas orientadas al apoyo de las pymes, contemplando los siguientes elementos del cuadro 13.

\section{Referencias}

[1] R. Hernández Sampieri, C. Fernández Collado \& M. Baptista Lucio. Metodología de la Investigación. México: Mc Graw Hill. 2014.

[2] C. Bueno. (2002). Administración y Dirección de Empresas de la Universidad a Distancia de Madrid. Recuperado el 04 de Junio de 2018, de https://blogs.udima.es/administracion-y-direccion-de-empresas/libros/ introduccion-a-la-organizacion-de-empresas-2/unidad-didactica-9-tamano-concentracion-y-crecimiento-dela-empresa/1-introduccion-concepto-de-tamano-y-crecimiento-de-la-empresa/

[3] Oxford. (2018). Recuperado el 04 de Junio de 2018, de https://es.oxforddictionaries.com/definicion/sector

[4] Alvarez \& Durán. (2009). Obtenido de Alvarez \& Durán, 2009.

[5] Oxford. (09 de 05 de 2018). Obtenido de https://es.oxforddictionaries.com/definicion/obstaculo

[6] Real Academia Española. (2017). Real Academia Española. Obtenido de http://dle.rae.es/?id=HT3X84s 